Original Article

\title{
Long-Term Outcomes of Cadaveric Lobar Lung Transplantation: An Important Surgical Option
}

\author{
Ilhan Inci, MD, ${ }^{1}$ Mace M. Schuurmans, MD,${ }^{2}$ Claudio Caviezel, MD,${ }^{1}$ Sven Hillinger, MD,${ }^{1}$ \\ Isabelle Opitz, MD ${ }^{1}$ Didier Schneiter, $\mathrm{MD},{ }^{1}$ and Walter Weder, $\mathrm{MD}^{1}$
}

\begin{abstract}
Background: Cadaveric lobar lung transplantation (L-LTx) is developed to overcome donor-recipient size mismatch. Controversial short- and long-term outcomes following L-LTx have been reported compared to full-sized lung transplantation (F-LTx). This study reports long-term outcomes after L-LTx.

Methods: We reviewed patients undergoing lung transplantation (LTx) between 2000 and 2016. The decision to perform L-LTx was made based mainly on donor-recipient height discrepancy and visual assessment of donor lungs. Predicted donor-recipient total lung capacity (TLC) ratio was calculated more recently. Primary outcome was overall survival. Results: In all, 370 bilateral LTx were performed during the study period, among those $250(67 \%)$ underwent F-LTx and $120(32 \%)$ underwent L-LTx, respectively. One- and 5-year survival rates were $85 \%$ vs. $90 \%$ and $53 \%$ vs. $63 \%$ for L-LTx and F-LTx, respectively ( $p=0.16)$. Chronic lung allograft dysfunction (CLAD)-free survival at 5 years was $48 \%$ in L-LTx vs. $51 \%$ in F-LTx recipients $(p=0.89)$, respectively. Age, intraoperative extracorporeal membrane oxygenation (ECMO) use, intensive care unit (ICU) stay, and postoperative renal replacement therapy (RRT) were significant prognostic factors for survival using multivariate analysis.

Conclusions: Overall survival and CLAD-free survival following L-LTx were comparable to F-LTx. Given the ongoing donor organ shortage, cadaveric L-LTx remains as an important resource in LTx.
\end{abstract}

Keywords: lobar lung transplantation, lung transplantation, survival, chronic rejection

\section{${ }^{1}$ Department of Thoracic Surgery, Zurich University Hospital, University of Zurich, Zurich, Switzerland \\ ${ }^{2}$ Division of Pulmonology, Zurich University Hospital, University of Zurich, Zurich, Switzerland}

Received: July 28, 2020; Accepted: October 13, 2020

Corresponding author: Ilhan Inci, MD, FEBTS. Department of Thoracic Surgery, University Hospital Zurich, Raemistrasse 100, CH-8091 Zurich, Switzerland

Email: ilhan.inci@usz.ch

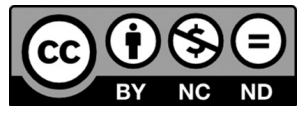

This work is licensed under a Creative Commons Attribution-NonCommercialNoDerivatives International License.

(C)2021 The Editorial Committee of Annals of Thoracic and Cardiovascular Surgery

\section{Introduction}

Lung transplantation (LTx) is an established treatment option for end-stage lung diseases. Donor shortage is still a problem and particularly threatens the small-sized and urgent candidates awaiting an appropriate organ donor. Some transplant centers developed an advanced operative technique called "downsizing" or "size reduced" LTx. ${ }^{1-7)}$ This includes peripheral wedge resections and lobar and split lung transplants to overcome size mismatch. Lobar LTx has been proposed to be an important surgical technique for those with smaller chest 
cavities and restrictive lung disease, leading to potentially fewer cases of waiting-list mortality. $\left.{ }^{4}\right)$ Due to its technical challenges, lobar lung transplantation (L-LTx) is not widely performed. ${ }^{8)}$

Although publications reporting on survival after L-LTx exist, there is no published data with special focus on the incidence of CLAD in L-LTx. Chronic lung allograft dysfunction (CLAD) is the major obstacle for long-term survival in LTx. ${ }^{9-15)}$ CLAD is reported to be the most common cause of death in recipients between 1 and 5 years after LTx. ${ }^{10-15)}$

The aim of this study is to investigate the long-term outcomes after L-LTx compared to full-sized lung transplantation (F-LTx).

\section{Materials and Methods}

Patient data are prospectively collected in our lung transplant program database starting from the first LTx conducted in November 1992. Between November 1992 and the end of September 2016, 485 patients underwent LTx at our center. Unilateral LTx, re-transplantations, and LTx performed before the year 2000 were excluded. After those exclusions, 370 patients remained for analysis and were included in the study. There were two groups, patients undergoing F-LTx $(\mathrm{N}=250)$ and patients undergoing L-LTx $(\mathrm{N}=120)$. The censor date for survival analysis was May 27, 2020. The decision to perform L-LTx was made prior to implantation based on donorrecipient height discrepancy and visual assessment of donor. We also calculated donor-recipient predicted total lung capacity (TLC) ratio (D-R pTLC). TLC is calculated according to standardized formula. ${ }^{16)}$ Donor lobectomies were performed on the back table prior to implantation. Primary graft dysfunction (PGD) grading is performed according to International Society for Heart and Lung Transplantation (ISHLT) consensus statement. ${ }^{17)}$ CLAD was defined as a persistent, obstructive decrease in forced expiratory volume in 1 second (FEV1) with at least $20 \%$ compared to the mean of the two best post-transplant values, in the absence of other identifiable causes. ${ }^{15)}$ Primary outcome was overall survival. Local research ethics committee approved the study (KEK Nr. 2019-00873).

\section{Statistical Analysis}

IBM SPSS version 25 (SPSS IBM, Armonk, New York, USA) was used for statistical analysis. Data are presented as median and interquartile range (IQR,
$25 \%-75 \%$ ). Categorical variables were compared using chi-square tests. Mann-Whitney test was used to compare continuous variables. Results of the multivariable linear or logistic regression analysis were adjusted for potential confounders such as recipient age, renal replacement therapy (RRT), and intraoperative extracorporeal membrane oxygenation (ECMO) use. Independent risk factors were identified by a step-wise backward regression analysis. Kaplan-Meier Method (log rank test) was used to calculate unadjusted survival rate and CLAD-free survival. A p value less than 0.05 was considered as the threshold for statistical significance.

\section{Results}

In this series, L-LTx constituted 32\% (120/370) of all LTx performed in this study cohort. Table 1 shows the recipient and donor characteristics of the study cohort. Gender and underlying diagnosis were statistically different between the two groups. D-R pTLC and donor and recipient height difference was also significantly different between the two groups.

There was a very significant correlation between D-R pTLC ratio and donor-recipient height difference ( $\mathrm{r}=$ $0.873, p=0.000$, Pearson correlation test).

Perioperative data are given in Table 2. L-LTx group needed more intraoperative ECMO use. Operation time and intensive care unit (ICU) stay were longer in the L-LTx group. In addition, L-LTx required more RRT, and the overall postoperative complication rate was higher. PGD Grade 2 and 3 at T48 was significantly higher in L-LTx recipients compared to F-LTx recipients.

In our series, 19 left upper lobe and 40 left lower lobe implantations were performed. On the right side, 25 middle and right lower lobe, 112 right upper lobe and right lower lobe and 11 right upper lobe and middle lobe implantations were performed.

We observed higher rates of tracheotomy, complications related to ECMO and critical illness neuromyopathy in L-LTx recipients (Table 3).

The rate of urgent listed recipients was $11.6 \%(\mathrm{~N}=14)$ in L-LTx and 7.2\% ( $\mathrm{N}=18)$ in F-LTx groups.

Early bronchial anastomosis complication occurred in one patient in the L-LTx group. Cartilage inversion in the anastomosis on the left side was corrected surgically after 24 hours following transplantation. As a late complication in three cases in the L-LTx group, bronchial stump insufficiencies (back table lower lobectomy, bronchial closure with stapler) leading to empyema were 
Table 1 Recipient and donor characteristics

\begin{tabular}{|c|c|c|c|}
\hline & L-LTx $(\mathrm{N}=120)$ & F-LTx $(\mathrm{N}=250)$ & $\mathrm{p}$ Value \\
\hline Age (years) & $53(43,58)$ & $58(50,60)$ & 0.1 \\
\hline $\operatorname{Sex}(F / M)$ & $71 / 49$ & $101 / 149$ & 0.001 \\
\hline Waiting list time (d) & $190(22,301)$ & $328(143,626)$ & 0.1 \\
\hline CRP at LTx & $12(6,29)$ & $3(2,6)$ & 0.2 \\
\hline Diagnosis & & & 0.001 \\
\hline $\mathrm{CF}$ & $46(38.3)$ & $81(32.4)$ & \\
\hline COPD & $21(17.5)$ & $89(35.6)$ & \\
\hline IPF & $38(31.6)$ & $37(14.8)$ & \\
\hline PAH & $5(4.1)$ & $15(6)$ & \\
\hline OTH & $10(8.3)$ & $28(11.2)$ & \\
\hline CMV (D/R) neg/pos & $30(25)$ & $57(22.8)$ & 0.3 \\
\hline BMI $\left(\mathrm{kg} / \mathrm{m}^{2}\right)$ & $20.5(18.3,29.4)$ & $23(20.5,26.8)$ & 0.3 \\
\hline R-Height (cm) & $165(159,172)$ & $173(168,182)$ & 0.001 \\
\hline R-Weight (kg) & $56(51,85)$ & $71(58,86)$ & 0.01 \\
\hline FEV1 preop (L) & $0.8(0.6,1.36)$ & $0.85(0.68,2.29)$ & 0.6 \\
\hline FEV1 preop (\%) & $30(25,44)$ & $30(20,65)$ & 0.1 \\
\hline \multicolumn{4}{|l|}{ Donor characteristics } \\
\hline $\operatorname{Sex}(\mathrm{F} / \mathrm{M})$ & $24 / 96$ & $116 / 134$ & 0.001 \\
\hline Age (years) & $54(45,58)$ & $53(45,71)$ & 0.6 \\
\hline Height $(\mathrm{cm})$ & $180(175,185)$ & $172(170,178)$ & 0.001 \\
\hline Weight $(\mathrm{kg})$ & $80(70,85)$ & $70(60,80)$ & 0.001 \\
\hline $\mathrm{PaO}_{2}-\mathrm{FiO}_{2}$ ratio $(\mathrm{kPa})$ & $47.1(36.8,62.6)$ & $44.7(34.7,58.8)$ & 0.3 \\
\hline D-R height difference $(\mathrm{cm})$ & $11.5(6,19)$ & $2(-3,7)$ & 0.001 \\
\hline D-R pTLC & $1.24(1.09,1.47)$ & $1.02(0.93,1.09)$ & 0.001 \\
\hline Donor type & & & 0.6 \\
\hline DBD & 115 & 236 & \\
\hline DCD & 5 & 14 & \\
\hline
\end{tabular}

All values are presented as $\mathrm{N}(\%)$ or median (Interquartile range: $25 \%-75 \%$ ). BMI: body mass index; CF: cystic fibrosis; CMV: cytomegalovirus; COPD: chronic obstructive pulmonary disease; CRP: C-reactive protein; d: days; D: donor; DBD: donation after brain death; DCD: donation after circulatory death; D-R pTLC: donor (D)-recipient (R) predicted total lung capacity (TLC) ratio; F: female; FEV1: forced expiratory volume in 1 second; $\mathrm{FiO}_{2}$ : fraction of inspired oxygen; F-LTx: full sized lung transplantation; IPF: idiopathic pulmonary fibrosis; kPa: kilopascal; L: liter; L-LTx: lobar lung transplantation; M: male; OTH: other; PAH: pulmonary arterial hypertension; $\mathrm{PaO}_{2}$ : arterial oxygen partial pressure; $\mathrm{R}$ : recipient

treated surgically using either omentum or muscle flap interposition. In the F-LTx group, we observed bronchial anastomosis complications in three cases, all of which were treated surgically early in the postoperative period.

Multivariate analysis of risk factors for mortality demonstrated that recipient age, intraoperative ECMO use, ICU stay, and RRT were risk factors for mortality in all patients (Table 4). Surgical procedure itself was not a risk factor.

$30-d(96 \%$ vs. $97 \%)$ and 90 -d survival rates (89\% vs. 94\%) were comparable between the groups (L-LTx vs. F-LTx).

One- and 5-year survival rates were $85 \%$ vs. $90 \%$ and $53 \%$ vs. $63 \%$ for L-LTx and F-LTx, respectively $(\mathrm{p}=$ 0.16) (Fig. 1).
Median time to CLAD was 1096.5 days (IQR, 319, 2432) for L-LTx and 1290 days (IQR, 531, 2444) for F-LTx groups. CLAD-free survival at 5 years was $48 \%$ in L-LTx and $51 \%$ in F-LTx recipients $(\mathrm{p}=0.89)$. During the study period, CLAD Stage 3 occurred in 54 recipients in L-LTx and in 110 in F-LTx recipients $(p=0.2)$.

\section{Discussion}

L-LTx is an additional option utilized in limited number of centers to overcome organ shortage, particularly in small recipients and urgent cases. ${ }^{1-8)}$ The gap between suitable donor lungs and the number of patients on the waiting list urged transplant centers to search for alternative strategies such as use of extended criteria donors organs, 
Table 2 Perioperative characteristics and outcome of the recipients

\begin{tabular}{|c|c|c|c|}
\hline & L-LTx & F-LTx & p-value \\
\hline Intraoperative ECMO & $76(63)$ & $108(43)$ & 0.001 \\
\hline Bridge to LTx & & & 0.3 \\
\hline ECMO & $11(9.1)$ & $14(5.6)$ & \\
\hline Intubated & $1(0.8)$ & $2(0.8)$ & \\
\hline Tracheotomy & $1(0.8)$ & - & \\
\hline ILA & $2(1.6)$ & $2(0.8)$ & \\
\hline PreTx-Postop ECMO & $7(5.8)$ & $5(2)$ & \\
\hline Intra-Postop ECMO & $9(7.5)$ & $6(2.4)$ & \\
\hline Postop ECMO & - & $6(2.4)$ & \\
\hline Total Op time (min) & $420(390,470)$ & $415(380,474)$ & 0.006 \\
\hline Intubation Time (d) & $1(1,15)$ & $1(1,1)$ & 0.08 \\
\hline ICU time $(\mathrm{d})$ & $12(3,31)$ & $2(1,3)$ & 0.01 \\
\hline RRT & $19(16)$ & $21(8)$ & 0.04 \\
\hline \multicolumn{4}{|l|}{ PGD } \\
\hline PGD2 T24 & $9(13)$ & $16(13)$ & 0.09 \\
\hline PGD2 T48 & $5(7)$ & $14(11)$ & 0.04 \\
\hline PGD2 T72 & $3(4)$ & $10(8)$ & 0.07 \\
\hline PGD3 T24 & $15(22)$ & $12(10)$ & 0.09 \\
\hline PGD3 T48 & $17(25)$ & $12(10)$ & 0.04 \\
\hline PGD3 T72 & $16(23)$ & $12(10)$ & 0.07 \\
\hline Surgical complication & $59(49)$ & $95(38)$ & 0.04 \\
\hline Re-exploration for bleeding & 16 & 18 & 0.1 \\
\hline 30-d mortality & $6(5)$ & $10(4)$ & 0.7 \\
\hline 90-d mortality & $13(11)$ & $17(7)$ & 0.7 \\
\hline CLAD free survival (d) & $1096.5(319,2432)$ & $1290(531,2444)$ & 0.89 \\
\hline
\end{tabular}

All values are presented as $\mathrm{N}(\%)$ or median (interquartile range: $25 \%-75 \%)$. CLAD: chronic lung allograft dysfunction; d: days; ECMO: extracorporeal membrane oxygenation; F-LTx: full-sized lung transplantation; ICU: intensive care unit; ILA: interventional lung assist device; L-LTx: lobar lung transplantation; Op: operation; PGD: primary graft dysfunction; Pre-Tx: pretransplantation; Postop: postoperative; RRT: renal replacement therapy

Table 3 Detailed list of complications occurred in the study groups

\begin{tabular}{lccc}
\hline & L-LTx & F-LTx & $\mathrm{p}$ Value \\
\hline Tracheotomy & $41(34)$ & $51(20.4)$ & 0.01 \\
Thoracic Hernia & $2(1.6)$ & $8(3.2)$ & 0.5 \\
Lymphocele & $9(7.5)$ & $13(5.2)$ & 0.3 \\
Phrenic nerve injury & $1(0.8)$ & $4(1.6)$ & 0.4 \\
Hemothorax & $24(20)$ & $24(9.6)$ & 0.1 \\
Pleural effusion & $6(5)$ & $21(8.4)$ & 0.1 \\
Pneumothorax & $4(3.3)$ & $12(4.8)$ & 0.1 \\
Bronchus anastomotic complications (overall) & $4(3.3)$ & $3(1.2)$ & 0.15 \\
- Early & $1(0.8)$ & $3(1.3)$ & \\
- Late* & $3(2.5)$ & - & 0.5 \\
Abdominal complications & $8(6.6)$ & $26(10.4)$ & 0.02 \\
Critical illness neuromyopathy & $12(10)$ & $9(3.6)$ & 0.01 \\
ECMO complications & $13(10.8)$ & $8(3.2)$ & \\
\hline
\end{tabular}

All values are presented as $\mathrm{N}(\%)$. *Bronchus anastomotic complication that occurred after discharge of the patient. ECMO: extra-corporeal membrane oxygenation; F-LTx: full sized lung transplantation; L-LTx: lobar lung transplantation 
Table 4 Multivariate analysis of risk factors for mortality

\begin{tabular}{lccc}
\hline Variable & Relative risk & $95 \%$ CI & p Value \\
\hline Recipient age & 1.022 & $1.013-1.032$ & 0.001 \\
Intraoperative ECMO use & 1.34 & $1.010-1.777$ & 0.04 \\
ICU stay & 1.01 & $1.004-1.016$ & 0.003 \\
RRT & 1.66 & $1.052-2.620$ & 0.03 \\
\hline
\end{tabular}

ECMO: extra-corporeal membrane oxygenation; ICU: intensive care unit; RRT: renal replacement therapy

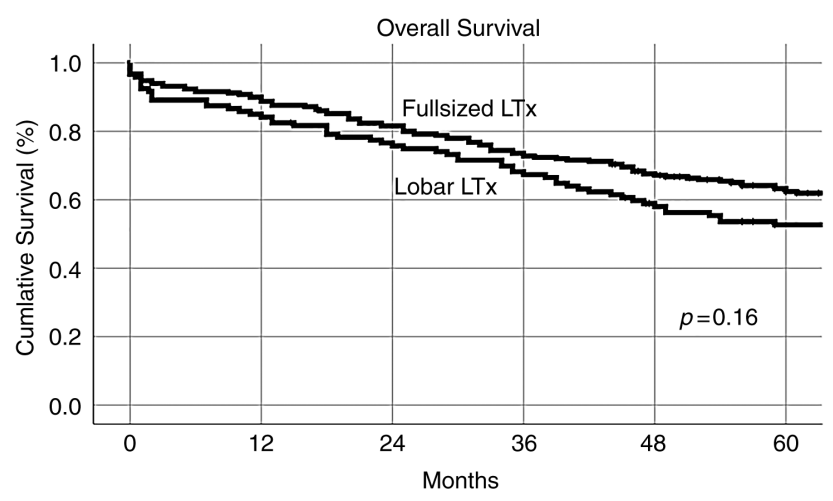

Fig. 1 Kaplan-Meier survival curve showing the overall survival between F-LTx and L-LTx. One- and 5-year survival rates were comparable between the two study groups $(\mathrm{p}=$ 0.16). F-LTx: full-sized lung transplantation; L-LTx: lobar lung transplantation

utilization of donation after circulatory death (DCD) donors, assessment and reconditioning of the so-called "marginal" lung in ex vivo lung perfusion system. ${ }^{18-21)}$

In our center, the rate of L-LTx consists one-third of our transplant population. Compared to series from Vienna, which reported 14\% L-LTx, ours is approximately two times more than Vienna Group. ${ }^{2)}$ One explanation might be the $34 \%$ rate of CF recipients and when we add the fibrosis recipients, this ratio goes up to $70 \%$ of our total cohort. As expected, these patients are generally small or they have a restricted thorax cavity (fibrosis patients). In Switzerland, we have patient-based allocation (Swiss Organ Allocation System [SOAS]) since May 2007. Before May 2007, the allocation system was center based. In both of those systems, there are no limitations for donor size during listing. In this system, we have "Urgent" status, which gives the recipient priority to get the first available donor lung, such as bridged to transplantation on ECMO and/or intubated patient. In our series, urgent-listed patients in L-LTx group were higher than F-LTx group.

L-LTx as a technique is not new and has been first published by Bisson et al. ${ }^{22}$ in the early 1990s. Other than to adapt a large size graft for a small recipient, this method also gives the opportunity to resect a lobe due to an unexpected pathology, such as total consolidated lung.2)

L-LTx is a technically challenging procedure requiring an experienced surgeon to perform a lobectomy on the back table. ${ }^{1,2)}$

Decision when to perform L-LTx differs among the centers. Donor-recipient difference or ratio in body weight and height, ${ }^{1,23,24)}$ chest circumference and chest $\mathrm{X}$-ray vertical and transverse dimensions, ${ }^{25}$ ) and the use of donor and recipient TLC for optimal size matching have been reported. ${ }^{2,26,27)}$ Slama and co-workers from Vienna reported that a TLC size discrepancy of less than $20 \%$ could be corrected by wedge resection alone, whereas a size reduction of $20 \%-60 \%$ requires L-LTX. ${ }^{2}$ However, final decision is made during the LTx procedure. ${ }^{1,2)}$ Although the international standard in most centers for donor-recipient size matching is based on D-R pTLC ratio, ${ }^{8)}$ in our center we mainly use height difference between donor and recipient. We also calculate pTLC ratio for our databank prospectively. As we demonstrated a significant correlation between donorrecipient height difference and D-R pTLC ratio, we believe that it is also possible and reliable to decide size mismatch with donor-recipient height difference.

ECMO use has been recommended to prevent reperfusion injury of the first-transplanted lobe. ${ }^{1-6)}$ The utilization rate of ECMO in published series varied between $32 \%$ and $70 \% .^{2-5}$ Despite the use of intraoperative ECMO, the occurrence rate of PGD rate in published reports could be as high as 54\%.,2,5) We observed $23 \%$ PGD 3 at T72 in L-LTx group. As the etiology of PGD is multifactorial, it is difficult to speculate only with the type of surgery combined with intraoperative ECMO use for high incidence of PGD in those series. In our series, we used ECMO in 63\%. A higher intraoperative ECMO use rate might be expected, but when the first side is fullsized transplantation and only the second side an L-LTx, 
the ECMO might be omitted. For bilateral L-LTx, ECMO implementation is recommended at the beginning of the transplantation. ${ }^{2)}$ In addition, routine practice of implanting the second lobe on ECMO to avoid over perfusion of the first implanted lobe has been reported.6)

Technical complications, such as bronchial anastomotic problems, kinking of vascular anastomoses, and remaining pleural dead space might occur. ${ }^{1,2)}$ The rate of bronchial anastomotic complications was $5.5 \%$ and $13 \%$, respectively, in the two recent series reporting L-LTx. ${ }^{3,23)}$ Early anastomotic problems occurred only in one patient in our L-LTx Group, which was corrected surgically 24 hours following the transplantation. The other three cases had bronchus stump insufficiency leading to empyema, all of which occurred late and after discharge of the recipients from the hospital. All of these cases were treated surgically by decortication and covering the stump with either omentum or thoracic muscle. Although all lobar combinations can be used in L-LTx, it is extremely important not to leave a bronchus stump by resecting the right lower lobe for implantation of the upper and middle lobes. ${ }^{2,4}$ On the left side, we also recommend not to leave a bronchus stump with stapler to make the anastomosis main-to-main bronchus: Lobar bronchus should be implanted to the recipient main bronchus.

Overall survival rates from different centers showed comparable results comparing L-LTx and F-LTx groups. ${ }^{4,5)}$ However, the Vienna Group, in their recent publication including 138 recipients, reported inferior survival in the L-LTx group compared to the F-LTx group. ${ }^{2)}$ We observed comparable survival between L-LTx and F-LTx groups as also reported from other centers. ${ }^{4,6)}$

CLAD is reported to be the most common cause of death in recipients between 1 and 5 years after LTx. ${ }^{9)}$ CLAD has an obstructive phenotype known as bronchiolitis obliterans syndrome (BOS). ${ }^{10,11)}$ PGD is one of the risk factors for the development of CLAD. ${ }^{15)}$ Increased rate of PGD has been reported from some centers after

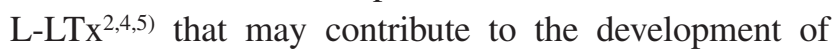
CLAD. ${ }^{15)}$ We observed a higher PGD Grade 2 and 3 at T48 in L-LTx recipients compared to F-LTx recipients. Despite high PGD grade observed in L-LTx group, CLAD-free survival was comparable between the two groups.

One of the limitations of our study is that we did not have the phenotypes of CLAD for the groups. The other limitation might be that we did not calculate the D-R pTLC ratio routinely at the beginning of the study.
In conclusion, in our series, overall and CLAD-free survival following L-LTx was comparable to F-LTx. Given the ongoing donor organ shortage, cadaveric L-LTx in our hands remains a viable option, in particular, for small and urgently listed LTx candidates.

\section{Disclosure Statement}

No conflicts of interest to disclose for any authors.

\section{References}

1) Inci I, Schuurmans MM, Kestenholz P, et al. Longterm outcomes of bilateral lobar lung transplantation. Eur J Cardiothorac Surg 2013; 43: 1220-5.

2) Slama A, Ghanim B, Klikovits T, et al. Lobar lung transplantation-is it comparable with standard lung transplantation? Transpl Int 2014; 27: 909-16.

3) Marasco SF, Than S, Keating D, et al. Cadaveric lobar lung transplantation: technical aspects. Ann Thorac Surg 2012; 93: 1836-42.

4) Shigemura N, D'Cunha J, Bhama JK, et al. Lobar lung transplantation: a relevant surgical option in the current era of lung allocation score. Ann Thorac Surg 2013; 96: 451-6.

5) Mitilian D, Sage E, Puyo P, et al. Techniques and results of lobar lung transplantations. Eur J Cardiothorac Surg 2014; 45: 365-9; discussion 369-70.

6) Stanzi A, Decaluwe H, Coosemans W, et al. Lobar lung transplantation from deceased donors: a valid option for small-sized patients with cystic fibrosis. Transplant Proc 2014; 46: 3154-9.

7) Barnard JB, Davies O, Curry P, et al. Size matching in lung transplantation: an evidence-based review. J Heart Lung Transplant 2013; 32: 849-60.

8) Eberlein M, Reed RM, Chahla M, et al. Lobar lung transplantation from deceased donors: a systematic review. World J Transplant 2017; 7: 70-80.

9) Chambers DC, Cherikh WS, Goldfarb SB, et al. The International Thoracic Organ Transplant Registry of the International Society for Heart and Lung Transplantation: Thirty-fifth adult lung and heart-lung transplant report-2018; Focus theme: multiorgan Transplantation. J Heart Lung Transplant 2018; 37: 1169-83.

10) Kulkarni HS, Cherikh WS, Chambers DC, et al. Bronchiolitis obliterans syndrome-free survival after lung transplantation: an International Society for Heart and Lung Transplantation Thoracic Transplant Registry analysis. J Heart Lung Transplant 2019; 38: 5-16.

11) Verleden GM, Vos R, Verleden SE, et al. Survival determinants in lung transplant patients with chronic allograft dysfunction. Transplantation 2011; 92: 703-8.

12) Meyer KC, Raghu G, Verleden GM, et al. An international ISHLT/ATS/ERS clinical practice guideline: 
diagnosis and management of bronchiolitis obliterans syndrome. Eur Respir J 2014; 44: 1479-503.

13) Verleden GM, Raghu G, Meyer KC, et al. A new classification system for chronic lung allograft dysfunction. J Heart Lung Transplant 2014; 33: 127-33.

14) Sato M, Waddell TK, Wagnetz U, et al. Restrictive allograft syndrome (RAS): a novel form of chronic lung allograft dysfunction. J Heart Lung Transplant 2011; 30: 735-42.

15) Verleden SE, Vos R, Vanaudenaerde BM, et al. Chronic lung allograft dysfunction phenotypes and treatment. J Thorac Dis 2017; 9: 2650-9.

16) Christie JD, Carby M, Bag R, et al. Report of the ISHLT Working Group on Primary Lung Graft Dysfunction part II: definition. A consensus statement of the International Society for Heart and Lung Transplantation. J Heart Lung Transplant 2005; 24: 1454-9.

17) Stocks J, Quanjer PH. Reference values for residual volume, functional residual capacity and total lung capacity. ATS Workshop on Lung Volume Measurements. Official Statement of the European Respiratory Society. Eur Respir J 1995; 8: 492-506.

18) Kotecha S, Hobson J, Fuller J, et al. Continued successful evolution of extended criteria donor lungs for transplantation. Ann Thorac Surg 2017; 104: 1702-9.

19) Inci I, Hillinger $S$, Schneiter $D$, et al. Lung transplantation with controlled donation after circulatory death donors. Ann Thorac Cardiovasc Surg 2018; 24: 296-302.

20) Van Raemdonck D, Keshavjee S, Levvey B, et al. International Society for Heart and Lung Transplantation.
Donation after circulatory death in lung transplantation-five-year follow-up from ISHLT Registry. J Heart Lung Transplant 2019; 38: 1235-45.

21) Cypel M, Yeung JC, Liu M, et al. Normothermic ex vivo lung perfusion in clinical lung transplantation. $\mathrm{N}$ Engl J Med 2011; 364: 1431-40.

22) Bisson A, Bonnette P, el Kadi NB, et al. Bilateral pulmonary lobe transplantation: left lower and right middle and lower lobes. Ann Thorac Surg 1994; 57: 219-21.

23) Inci I, Irani S, Kestenholz $\mathrm{P}$, et al. Donor predicted post-operative forced expiratory volume in one second predicts recipients' best forced expiratory volume in one second following size-reduced lung transplantation. Eur J Cardiothorac Surg 2011; 39: 115-9.

24) Aigner C, Mazhar S, Jaksch P, et al. Lobar transplantation, split lung transplantation and peripheral segmental resection-reliable procedures for downsizing donor lungs. Eur J Cardiothorac Surg 2004; 25: 179-83.

25) Aigner $C$, Jaksch $P$, Taghavi $S$, et al. Donor total lung capacity predicts recipient total lung capacity after size-reduced lung transplantation. J Heart Lung Transplant 2005; 24: 2098-102.

26) Eberlein M, Reed RM, Bolukbas S, et al. Lung size mismatch and survival after single and bilateral lung transplantation. Ann Thorac Surg 2013; 96: 457-63.

27) Eberlein M, Reed RM, Permutt S, et al. Parameters of donor-recipient size mismatch and survival after bilateral lung transplantation. J Heart Lung Transplant 2012; 31: 1207-13.e7. 